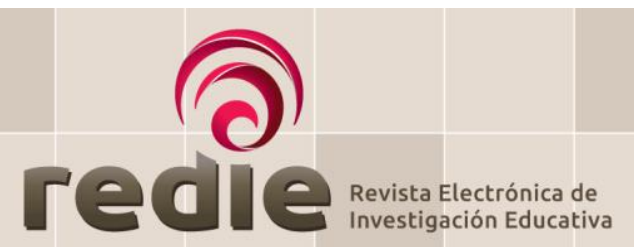

ISSN: $1607-4041$

https://redie.uabc.mx

Vol. 23, 2021/e04

\title{
Migración de retorno en México y su bilingüismo. Revisión de la literatura 2006-2018 1
}

\section{Return Migration in Mexico and its Bilingualism. A Literature Review 2006-2018}

Kalinka Velasco Zárate (*) https://orcid.org/0000-0001-6610-3502

(*) Universidad Autónoma Benito Juárez de Oaxaca

(Recibido: 22 de diciembre de 2019; Aceptado para publicación: 1 de abril de 2020)

Cómo citar: Velasco, K. (2021). Migración de retorno en México y su bilinguismo. Revisión de la literatura 2006-2018. Revista Electrónica de Investigación Educativa, 23, e04, 1-12. https://doi.org/10.24320/redie.2021.23.e04.3719

\section{Resumen}

Se presenta una revisión de la literatura sobre los estudiantes migrantes de retorno en el sistema educativo en México, la cual incluyó artículos de investigación y otros textos académicos disponibles publicados en el período 2006-2018. La revisión permitió identificar los métodos investigativos y temáticas de estudio: una tipología de estos estudiantes basada en variables como edad, nacionalidad y estatus migratorio, condiciones al ingreso y las necesidades surgidas durante su estancia en el contexto educativo. También emergió un perfil educativo, con base en información sobre sus características psicológicas, experiencias lingüísticas y escolares previas, así como aspectos relacionados con la convivencia escolar e integración social. Aunque la investigación existente es útil para conocer algunas características y necesidades al retorno, aún son necesarios estudios que indaguen aspectos como la relación bilingüismo-desarrollo académico en los distintos niveles educativos.

Palabras clave: migración de retorno, bilingüismo, política lingüística, integración escolar

\section{Abstract}

A literature review is presented about the returning migrant students in the educational system in Mexico, which included both research papers and other types of academic texts, available from 2006 to 2018, and around this population. The revision allowed to identify the research methods and the thematic issues: a typology of these students based in variables such as age, nationality and migration status, the conditions upon the access to and the needs that arise during their permanence in the educational context. Also an educational profile emerged, based on information about their psychological traits, their previous linguistic and schooling experiences, as well as aspects related with the school coexistence and the social integration. Although the existent research is useful to know their characteristics and needs upon the return, studies that inquire aspects such as the relationship bilingualism-academic development, in the different educational levels, are still scarce and thus necessary.

Keywords: return migration, bilingualism, language policy, school integration

\footnotetext{
${ }^{1}$ Estudio realizado gracias el apoyo de la UABJO, Facultad de Idiomas, Recurso PFCE 2018.
} 


\section{Introducción}

La presencia de estudiantes migrantes de retorno en las aulas del sistema educativo mexicano es un fenómeno relacionado con los flujos migratorios y los cambios ocurridos en las dinámicas económicas y políticas migratorias entre México y Estados Unidos de América (EUA) en las dos últimas décadas. Estos estudiantes han vivido y estudiado en contextos distintos al de origen, por lo que sus experiencias de vida, formación, conocimientos y habilidades están asociadas a su permanencia en el país extranjero. Entre esos conocimientos y experiencias está el posible desarrollo del bilingüismo, es decir, el conocimiento y manejo de más de un idioma (el de origen y el del lugar del contexto al que migran), como resultado de la interacción en distintos espacios de la vida diaria y escolar. Sin embargo, el fenómeno del retorno ha recibido mayor atención respecto al impacto del regreso al país de origen, en relación con variables socioeconómicas y su integración al contexto social y, en menor medida, a la presencia de la población migrante en las aulas, a sus necesidades y desarrollo académico. Este artículo tiene como propósito documentar la investigación realizada en torno a la situación que prevalece en el contexto educativo al que ingresa la población bilingüe de retorno en México y en Oaxaca para identificar las temáticas abordadas, los métodos y hallazgos para plantear posibles líneas de investigación que permitan atender mejor sus necesidades.

\subsection{Bilingüismo}

El bilingüismo es un término complejo, y por ello un constructo con distintas dimensiones como la lingüística, cognitiva, psicológica y social que considerar, cuando se trata de definir y de investigar (Baker y Wright, 2017; Edward, 2014). El término se refiere a tener conocimiento y habilidades en más de una lengua, aunque las definiciones varían respecto al grado de dominio de una u otra. Una definición clásica de bilingüismo considera el grado de dominio (conocimiento y uso) que se hace de dos lenguas mostrando el control a nivel nativo de estas (Baker y Wright, 2017; Edward, 2014); esto implica estar expuesto a dos lenguas desde muy temprana infancia y haber crecido usando ambas por igual en la comprensión y la producción oral (Serratrice, 2014). Dado que no todas las personas que habitan en un contexto en donde se usa más de una lengua pueden hacer uso de éstas en igual grado, otras definiciones de bilingüismo consideran tanto diferentes niveles del dominio de las lenguas como usos diferenciados de éstas. Así, August y Shanahan (2006) consideran que una persona es bilingüe cuando habla una lengua adicional, distinta a la del grupo social en que está inmersa; puede hablar la lengua de origen como hablante nativo y haber desarrollado un nivel de competencia en la segunda lengua en similar o distinto grado, y en un momento dado, el grado de habilidad varía dependiendo del contexto de uso y a lo largo del tiempo.

En general, el estudio del bilingüismo considera factores como: etapas de su desarrollo, grado de dominio, habilidades desarrolladas, edad, condiciones y momentos de exposición a los idiomas, así como aspectos sociolingüísticos como contacto, dominios y propósitos de uso, mantenimiento y pérdida; también se puede agregar el factor cultura, para hablar de bilingüismo bicultural, multicultural o de aculturación (Baker y Wright, 2017; Edward, 2014; Goto-Butler, 2014; Wei, 2014). Por último, uno de los factores asociados al desarrollo del bilingüismo son los procesos migratorios.

\subsection{Migración de retorno en México}

Los estudios de determinantes sociodemográficas que han tenido como interés a la población de retorno sitúan la migración histórica de mexicanos a los EUA en dos tipos: migrantes con residencia fija (settlers) y los migrantes circulares (sojournes), quienes por empleos temporales transitan hacia ese país impactando en la economía de sus comunidades y familias de origen a través de las remesas; además, mantienen redes sociales donde movilidad física, economía, emociones y cultura de individuos y familias de ambos lugares se involucran (Smith 2006; Canales, 2001). Así, la economía es una de las principales causas relacionadas con la migración circular de mexicanos a EUA que se registra en la década de los setenta, intensificándose en los ochenta, y que incluye a indocumentados jóvenes, solteros, de baja escolaridad y de extracción rural (Canales, 2001), o con escolaridad media y de extracción urbana en busca de oportunidades laborales (Vargas y Camacho, 2015). La Amnistía de 1986 permitió que alrededor de 2 millones de mexicanos que 
residían en EUA regularizaran su situación migratoria (Zúñiga, 2013).

A finales de los noventa y durante la primera década del siglo XXI la dirección del flujo migratorio cambió, como consecuencia de la crisis económica y las nuevas políticas migratorias en los EUA (Aguilar, 2012, 2015; Canales, 2001; Vargas y Camacho, 2015; Zúñiga, 2013), como resultado, aumentó el número de migrantes de retorno a México.

El perfil de las personas en el flujo de retorno de la migración internacional se reconfiguró e incluyó no sólo a hombres adultos sino a jóvenes, mujeres y niños (Aguilar, 2012; 2015; Alvarado y Reyes, 2017; Santos et al., 2010; Valdéz et al., 2018; Zúñiga, 2013); de ser un fenómeno individual pasó a involucrar a las familias y redes sociales, ubicadas en diferentes lugares; así, un proceso individual y homogéneo pasó a ser social y heterogéneo (Valdéz et al., 2018; Vargas y Camacho, 2015). Además, el retorno no puede considerarse definitivo porque en períodos cortos ha sido lento pero gradual, pues en 2007 se registraron 12.8 millones de mexicanos emigrantes en los EUA comparados con 11.7 millones en 2014 (Gonzalez-Barrera, 2015; Jensen et al., 2017), entre los que se encuentran niños y adolescentes binacionales, que sumaron alrededor de 500000 personas en el Censo 2010 (Zúñiga, 2013). Algunas razones para el regreso reportadas por los migrantes son: reunificación familiar, crisis económica, deportación como resultado del endurecimiento de las reglas para permanecer o para cruzar la frontera, enfermedades, perspectivas de continuar los estudios universitarios y el cumplimiento de cargos por sistemas normativos (Aguilar, 2012; Alvarado y Reyes, 2017; Camacho y Vargas, 2017; Cortez et al. 2015; Galindo, 2016; Nava et al., 2017; Santos et al., 2010).

En general, a partir del 2005, entre los migrantes retornados a México predominan menores en edad escolar y jóvenes adultos que traen consigo otras experiencias de formación, conocimientos e idioma, por lo que brindarles atención puede ser considerado un desafío o una oportunidad para el sistema educativo en México (Nava, et al., 2017; Valdéz et al., 2018; Zúñiga, 2013). Respecto a Oaxaca, entidad emergente en este fenómeno (Vargas y Camacho, 2015), en la distribución de menores retornados por Estado de residencia y según lugar de nacimiento hay más niños nacidos en EUA que en México (la distribución porcentual en el año 2000 fue 2.6 y en el 2010 de 3.6 (Aguilar, 2012, p. 24). En enero de 2017, alrededor de 7157 migrantes oaxaqueños fueron repatriados, entre los cuales hay jóvenes que nacieron en EUA, con familias de origen mexicano o que fueron llevados a edades tempranas (Huerta et al., 2017), siendo la región de los Valles Centrales la de mayor recepción de estudiantes de retorno (Alvarado y Reyes, 2017).

\section{Método}

Ante la necesidad de conocer el estado de la investigación sobre los estudiantes migrantes de retorno en el contexto educativo, y en particular, sobre el estudio de su bilingüismo, se llevó a cabo una revisión de la literatura. Los estudios se encontraron haciendo búsquedas de artículos en bases de datos dentro de los recursos disponibles en el Consorcio Nacional de Recursos de Información Científica y Tecnológica (CONRICYT) de la página del Consejo Nacional de Ciencia y Tencnología (CONACYT), y en el buscador Google Scholar de mayo a octubre de 2018; una nueva búsqueda en abril de 2020 no arrojó nuevos resultados.

Para realizar las búsquedas las palabras clave fueron: Returnees/retornados, transnationals/trasnacionales, migración de retorno, México, deportation/deportación, escuela, inserción escolar, y bilingüe/bilingual. Se excluyeron de los resultados los estudios no relacionados con este tipo de migración en el contexto educativo mexicano y se obtuvieron 17 documentos con un rango de publicación del 2006 al 2018: 9 artículos de investigación de revistas arbitradas por pares y 6 documentos más, entre los que se encuentran ponencias y actas de congresos, capítulos de libros y artículos en revistas no arbitradas. Se incluyeron también 2 documentos (Tesis y capítulo de libro) fuera de línea, que abordan el tema de la migración de retorno en el contexto oaxaqueño. La lectura activa y un proceso de codificación abierta con apoyo de matrices (Hernández et al., 2006) permitieron analizar y contrastar el contenido en términos de las temáticas abordadas, las características de los estudiantes, y los contextos y niveles educativos para obtener un panorama reciente de la investigación realizada en México, y en Oaxaca en particular, sobre este tipo de migración, su impacto en el contexto educativo y en relación con el bilingüismo. 


\section{Resultados}

La tabla 1 muestra los estudios incluidos en la revisión de la literatura (2006-2018); los estudios se presentan por nivel educativo y en orden cronológico según su publicación.

Tabla 1. Estudios sobre la Migración de retorno en el contexto educativo

\begin{tabular}{|c|c|c|c|}
\hline Estudio & Foco del estudio & Entidad & Método \\
\hline \multicolumn{4}{|c|}{ Nivel Kinder-Primaria } \\
\hline $\begin{array}{l}\text { 1. Smith } \\
(2006)\end{array}$ & $\begin{array}{l}\text { Delinear política lingüística: atención a } \\
\text { derechos humanos y lingüísticos en las } \\
\text { escuelas al reincorporarse a éstas. }\end{array}$ & $\begin{array}{l}\text { Puebla y escuelas de } \\
\text { educación básica en } \\
\text { zonas de alto índice } \\
\text { de migración. }\end{array}$ & $\begin{array}{l}\text { Análisis cualitativo de casos de } \\
\text { retornados. }\end{array}$ \\
\hline $\begin{array}{l}\text { 2. Santos } \\
\text { et al. } \\
(2010)\end{array}$ & $\begin{array}{l}\text { Estudiar el impacto del retorno a } \\
\text { México de niños nacidos en EUA a su } \\
\text { ingreso a escuelas de educación } \\
\text { básica. }\end{array}$ & San Luis Potosí & $\begin{array}{l}\text { Diagnóstico, con enfoque } \\
\text { inductivo-descriptivo. }\end{array}$ \\
\hline $\begin{array}{l}\text { 3. Zúñiga } \\
\text { (2013) }\end{array}$ & $\begin{array}{l}\text { Dimensionar el volumen de alumnos } \\
\text { que han participado en la migración } \\
\text { internacional con datos de } 4 \text { lugares y } \\
\text { proponer } 3 \text { subpoblaciones de } \\
\text { alumnos. }\end{array}$ & $\begin{array}{l}\text { Nuevo León, } \\
\text { Zacatecas, Puebla, } \\
\text { Jalisco }\end{array}$ & Encuestas, entrevistas. \\
\hline $\begin{array}{l}\text { 4. Jensen } \\
\text { et al. } \\
(2017)\end{array}$ & $\begin{array}{l}\text { Identificar tendencias demográficas } \\
\text { básicas de la población infantil de } \\
\text { retorno y analizar las dimensiones de } \\
\text { calidad en el aula del preescolar. }\end{array}$ & Aguascalientes & $\begin{array}{l}\text { Análisis de datos del Censo 2010, } \\
\text { INEGI y de la Encuesta Intercensal } \\
2015, \text { INEGI y análisis de video de } \\
\text { observaciones de clases en } \\
\text { escuelas de primer ciclo educación } \\
\text { básica. }\end{array}$ \\
\hline $\begin{array}{l}\text { 5. Aguilar } \\
(2015)\end{array}$ & $\begin{array}{l}\text { Mostrar que la medición de la } \\
\text { integración de la migración de retorno } \\
\text { de niños y jóvenes debe considerar } \\
\text { variables individuales como edad y } \\
\text { lugar de nacimiento para comprender } \\
\text { el proceso de integración y el } \\
\text { desempeño escolar. }\end{array}$ & $\begin{array}{l}\text { Todos los menores } \\
\text { migrantes } \\
\text { retornados de 5-17 } \\
\text { años. }\end{array}$ & $\begin{array}{l}\text { Análisis de datos } \\
\text { sociodemográficos y propuesta de } \\
\text { modelo para análisis cuantitativo } \\
\text { que incluye variables individuales } \\
\text { como lugar de nacimiento y edad. }\end{array}$ \\
\hline $\begin{array}{l}\text { 6. Valdéz } \\
\text { et al. } \\
\text { (2018) }\end{array}$ & $\begin{array}{l}\text { Analizar problemáticas académicas } \\
\text { relacionadas con trámites } \\
\text { administrativos. }\end{array}$ & Sonora & $\begin{array}{l}\text { Estudio de caso, entrevistas a } \\
\text { directores de escuelas primarias. }\end{array}$ \\
\hline
\end{tabular}

\section{Nivel Secundaria}

7. Santos Véase el estudio $2 . \quad$ Véase el estudio $2 . \quad$ Véase el estudio 2.

et al.

(2010)

\begin{tabular}{|c|c|c|c|}
\hline $\begin{array}{l}\text { 8. Zúñiga } \\
\text { (2013) }\end{array}$ & Véase el estudio 3. & Véase el estudio 3. & Véase el estudio 3. \\
\hline $\begin{array}{l}\text { 9. Vargas y } \\
\text { Camacho } \\
(2015)\end{array}$ & $\begin{array}{l}\text { Analizar el rezago escolar en México } \\
\text { entre estudiantes de retorno nacidos } \\
\text { en México y en EUA. }\end{array}$ & $\begin{array}{l}3 \text { zonas de migración } \\
\text { Norte-tradicional, } \\
\text { Centro y Sureste. }\end{array}$ & $\begin{array}{l}\text { Análisis de variables } \\
\text { sociodemográficas basado en } \\
\text { datos INEGl } 2010 .\end{array}$ \\
\hline $\begin{array}{l}10 . \\
\text { Camacho y } \\
\text { Vargas }\end{array}$ & $\begin{array}{l}\text { Indagar la relación de capital } \\
\text { económico, humano-cultural y social } \\
\text { con el rezago escolar de los niños de }\end{array}$ & $\begin{array}{l}\text { Niños 8-15 años de } \\
\text { edad migrantes de } \\
\text { retorno a Baja }\end{array}$ & $\begin{array}{l}\text { Diseño Mixto: análisis multinomial } \\
\text { de datos del Censo } 2010 \text { y } \\
\text { entrevistas semiestructuradas. }\end{array}$ \\
\hline
\end{tabular}

(2017) retorno y sus experiencias de inserción

California y Tijuana 


\begin{tabular}{|c|c|c|c|}
\hline Estudio & Foco del estudio & Entidad & Método \\
\hline \multicolumn{4}{|c|}{ Nivel Medio Superior } \\
\hline $\begin{array}{l}\text { 11. Vargas } \\
\text { y Camacho } \\
(2015)\end{array}$ & Véase el estudio 9. & Véase el estudio 9 & Véase el estudio 9. \\
\hline $\begin{array}{l}12 . \\
\text { Alvarado y } \\
\text { Reyes } \\
(2017)\end{array}$ & $\begin{array}{l}\text { Analizar la convivencia escolar entre } \\
\text { los jóvenes de retorno temporal o } \\
\text { permanente. }\end{array}$ & Oaxaca & $\begin{array}{l}\text { Estudio de caso; observaciones, } \\
\text { entrevistas. }\end{array}$ \\
\hline \multicolumn{4}{|c|}{ Nivel Superior } \\
\hline $\begin{array}{l}\text { 13. Cortez } \\
\text { et al. } \\
(2015)\end{array}$ & $\begin{array}{l}\text { Examinar las experiencias de } \\
\text { migración de los estudiantes } \\
\text { universitarios de retorno y su relación } \\
\text { con las nuevas experiencias sociales y } \\
\text { educativas. }\end{array}$ & Sonora & $\begin{array}{l}\text { Estudio cualitativo; entrevistas, } \\
\text { observaciones, notas de campo. }\end{array}$ \\
\hline $\begin{array}{l}\text { 14. Galindo } \\
(2016)\end{array}$ & $\begin{array}{l}\text { Conocer las experiencias de los } \\
\text { estudiantes universitarios al retorno y } \\
\text { proponer acciones para facilitar su } \\
\text { integración. }\end{array}$ & Oaxaca & Entrevistas y narraciones (4 casos). \\
\hline $\begin{array}{l}\text { 15. Cortez } \\
\text { et al. } \\
(2017)\end{array}$ & $\begin{array}{l}\text { Analizar el efecto del modelo de } \\
\text { inmersión en el mantenimiento del } \\
\text { bilingüismo de jóvenes adultos } \\
\text { biculturales. }\end{array}$ & Sonora & $\begin{array}{l}\text { Metodología mixta longitudinal; } \\
\text { encuestas y entrevistas en } \\
\text { profundidad. }\end{array}$ \\
\hline $\begin{array}{l}\text { 16. Huerta } \\
\text { et al. } \\
\text { (2017) }\end{array}$ & $\begin{array}{l}\text { Reportar la propuesta de intervención } \\
\text { basada en la tutoría entre iguales en } \\
\text { un programa de formación de } \\
\text { docentes de idiomas. }\end{array}$ & Oaxaca & $\begin{array}{l}\text { Intervención en el aula de tutorías } \\
\text { entre iguales para la mejora del } \\
\text { inglés y español. }\end{array}$ \\
\hline $\begin{array}{l}\text { 17. Nava et } \\
\text { al. (2017) }\end{array}$ & $\begin{array}{l}\text { Conocer las competencias de } \\
\text { literacidad académica en español e } \\
\text { inglés, y de otras áreas disciplinares. }\end{array}$ & Tlaxcala & $\begin{array}{l}\text { Estudio exploratorio mixto } \\
\text { (Cuestionarios, entrevista a } \\
\text { profundidad, observaciones de } \\
\text { clase). }\end{array}$ \\
\hline
\end{tabular}

\subsection{Temas investigados}

Los estudios indagan la situación de los estudiantes migrantes de retorno en estados de las regiones de altos y medianos índices de migración (Frontera Norte, Centro del país y Sureste): Aguascalientes, Baja California, Jalisco, Nuevo León, Oaxaca, Puebla, San Luis Potosí, Sonora, Tlaxcala y Zacatecas -incluyendo estudiantes y escuelas que comprenden estados dentro de una región migratoria o ciertos municipios en una entidad federativa, hasta estudios de casos.

De los datos sociodemográficos, experiencias, opiniones y observaciones reportados, se identificaron los siguientes temas sobre esta población: distintas definiciones que conforman una tipología de migrantes estudiantes de retorno, un perfil general del estudiante de retorno (en términos educativos y psicológicos), los tipos de bilingüismo que representan, los retos institucionales (administración, currículo, enseñanza) y para el sistema educativo en México, así como las recomendaciones para política educativa y lingüística que en su momento los autores plantearon.

\subsection{Definición y tipología}

La definición de este tipo de migración es heterogénea, dada la variedad de condiciones y experiencias de este tipo de migrantes. Una definición general es la de Aguilar (2015):

Un movimiento migratorio entre fronteras nacionales (...) de personas que nacieron en el país al cual están inmigrando (...), en este caso específico son aquellas personas que nacieron en México, 
emigraron a los Estados Unidos y regresaron a México después de haber radicado en Estados Unidos. (p. 247). ${ }^{2}$

Zúñiga (2013) considera necesario sumar a las variables lugar de nacimiento y nacionalidad, la edad al momento del retorno y lugar de la escolaridad para distinguir aspectos que las definiciones generales no muestran. En su definición, Zúñiga distingue la edad de los migrantes retornados, considerando como menor a quien no ha alcanzado la mayoría de edad (18 años), que puede haber migrado a EUA o haber nacido en ese país, pero en algún momento (y antes de ser considerado adulto) regresó a México.

En general, esos estudiantes comenzaron su escolaridad en México y continuaron en escuelas en EUA; si nacieron en EUA pueden tener la doble nacionalidad, por derecho de suelo o por sangre, por lo que se les considera binacionales a su regreso a México. Zúñiga (2013) ofrece la siguiente tipología del migrante retornado:

1. Trasnacionales: personas nacidas en México, quienes de niños o adolescentes migraron hacia los EUA con sus padres o al menos uno de ellos, y después de cierto período de residencia en EUA regresaron a México. En general, esos estudiantes comenzaron su escolaridad en México y continuaron en escuelas en EUA, por lo que transitaron en dos contextos educativos, pero todavía se identifican con su filiación mexicana.

2. Binacionales: niños nacidos en los EUA y que regresan a México con sus padres por primera vez en su vida (migrantes internacionales o inmigrantes), con experiencias de escolaridad en EUA.

Según Zúñiga (2013) los dos grupos no se excluyen, pues la siguiente situación también es posible:

3. Trasnacional-binacional: nacidos en los EUA pero que antes de la edad escolar regresaron a México.

Otros términos que se relacian con el contexto educativo y cultural son:

4. Binacionales-trasnacionales: menores retornados de constante movilidad, ya que pueden regresar a Estados Unidos o a México por períodos cortos, afectando su escolaridad, y quienes se encuentran atrapados entre dos mundos o pertenecen a dos culturas (Santos et al., 2010, p. 5).

5. Retornados adultos jóvenes (mayores de 18 años; Dreamers): jóvenes en edad de estudiar la universidad, quienes regresan con o sin sus padres, y a veces de manera forzada, para continuar sus estudios universitarios (Cortez et al., 2015, 2017; Nava et al., 2017). Estos estudiantes originalmente migraron con sus familias a EUA cuando niños, crecieron y estudiaron en escuelas públicas y aprendieron a hablar inglés con acento de estadounidense nativo. Se les llama Dreamers porque la falta de un número de seguridad social evitó su ingreso a la universidad, o no tuvieron garantías de igualdad de oportunidades por su estatus migratorio a pesar del tiempo vivido en los EUA. ${ }^{3}$

\subsection{Perfil educativo}

Primero, Santos et al. (2010) y Zúñiga (2013) consideran que se trata de estudiantes menores y mayores de edad con experiencias escolares diversas por formarse en currículos basados en modelos pedagógicos, con contenidos y valores distintos según estados y distritos escolares, aunado al hecho de que en EUA el sistema educativo está descentralizado. También conocieron docentes con características y niveles de formación distintos, y estudiaron en escuelas con diferentes o similares condiciones de infraestructura a

\footnotetext{
${ }^{2}$ Aguilar (2015) propone redefinir el concepto para referirse a toda la población de entre 5 y 17 años que haya vivido en los EUA, cuando su país de nacimiento sea México o los EUA, en hogares donde el jefe(a) es mexicano.

${ }^{3}$ Camacho y Vargas (2017) vinculan a este tipo de migración la desintegración familiar al haber menores separados de los padres, que viven con otros familiares o bajo la tutela de la asistencia social.
} 
las que regresan (Cortez et al., 2015; Santos et al., 2010). En algunos casos no siguen los mismos ritmos o patrones de aprendizaje que el resto de sus compañeros, por tratarse de estudiantes de alto rendimiento con habilidades para la lectoescritura en inglés y matemáticas que transfieren a los estudios en el país al que migran (Cortez et al., 2015), o por ser estudiantes con necesidades de apoyo, como en la lectoescritura académica en español como L2, relacionado con un bilingüismo desbalanceado (Nava et al., 2017, pp. 125126).

Segundo, la movilidad constante de EUA a México de los menores migrantes afecta su historia escolar, pues permanecer por estancias cortas da como resultado trayectorias escolares insatisfactorias e incompletas. También, en muchos casos, hay disparidad en la correspondencia entre edad y grado escolar respecto el sistema mexicano (Santos et al., 2010; Vargas y Camacho, 2015). Camacho y Vargas (2017) encontraron que los niños y adolescentes migrantes en zonas fronterizas de alta migración se ubican principalmente en zonas rurales; de estos, los adolescentes tienen mayor rezago escolar (destiempo en la inserción escolar, trayectorias truncas, inasistencias) que los de primaria, debido a la interrupción de estudios para trabajar. Para ambos, los bajos capitales cultural-humano de los padres (nivel escolar) y el social (redes sociales en el lugar de retorno) inciden negativamente en la asistencia y permanencia en la escuela.

También entre los estudiantes de bachillerato el rendimiento escolar puede verse perjudicado por el estudio y el trabajo simultáneo (Alvarado y Reyes, 2017). Aunque los binacionales suelen tener un mayor capital social y financiero, incrementando así las probabilidades del buen término de sus estudios (Vargas y Camacho, 2015), esto no es el caso de los adultos jóvenes (Dreamers), quienes logran alcanzar niveles de escolaridad más altos que sus padres, pero al retorno involuntario a México un débil capital social incide de forma negativa en el logro de sus metas académicas (Cortez et al., 2017).

Por último, Aguilar (2015) propone una metodología basada en un análisis intergeneracional para conocer la integración de la migración de retorno a la sociedad receptora, donde los indicadores de la integración sean el desarrollo económico y el desempeño escolar (escolaridad-educación); así, variables individuales como edad y lugar de nacimiento, entre otras, son importantes para comprender el impacto de la movilidad de los nacidos en EUA en sus trayectorias escolares y en su desempeño escolar.

\subsection{Características psicológicas}

Cortez et al. (2015) muestran que los estudiantes adultos jóvenes tienen la capacidad para resignificar sus experiencias adversas; a pesar de las tribulaciones, algunos desarrollan resiliencia (Cortez et al., 2017) y se asumen como agentes en la toma de decisiones que les afectan, sobreponiéndose a los múltiples procesos de cambio y malas experiencias (Cortez et al., 2015). Una estrategia para la resignificación es la construcción de una identidad a través de imaginarios relacionados con el futuro, donde se ven como personas capaces y con acceso a estudios universitarios, posibilidades de vida y empleo, incluso en contextos distintos al de retorno; frente a lazos económicos y sociales debilitados sus conocimientos del inglés y la cultura del lugar son usados como recurso para obtener un empleo y estudiar (Nava et al., 2017).

Por su parte, Galindo (2016) indaga las experiencias de estudiantes universitarios que migraron siendo niños, y centra su interés en aspectos emocionales como la ansiedad (que permea a la llegada al país receptor) y el proceso de aculturación y aprendizaje de la L2 (inglés/español), y la tristeza, nostalgia y shock cultural que acompañan el retorno y el proceso de enculturación; respecto al idioma, el nivel que pudieron desarrollar del español L1/L2, antes o durante la estancia en EUA, incide en la socialización y desempeño académico al retorno, facilitándose estos para los estudiantes de docencia del inglés. Nava et al. (2017, pp.125-133) observan también que la formación sociocultural en contextos distintos dificulta afianzar las identidades, coincidiendo parcialmente con Galindo (2016), respecto a las ventajas que representa su conocimiento del inglés para su formación como docentes de este idioma, pues también pueden carecer del metalenguaje y de conocimientos léxico-gramaticales necesarios para esta profesión. 


\subsection{Tipos de bilingüismo y su mantenimiento}

Smith (2006) señala que los menores estudiantes migrantes de retorno (6-15 años, nacidos y no nacidos en EUA), pueden representar diferentes tipos de bilingüismo y características sociolingüísticas (como el uso de variedades consideradas no estándar) del inglés y del español, relacionadas con las condiciones de contexto y casa, bajos grados de escolaridad, la influencia de redes sociales, modelos de instrucción en las escuelas en donde estudiaron y las trayectorias de migración.

Vargas y Camacho (2015, p. 170) registran que la mayoría de los menores entre 11-15 años de inmigración reciente de los EUA son bilingües en algún grado en inglés y español, pero también pueden ser hablantes de una lengua indígena ( $0.4 \%$ de estudiantes vs. $96 \%$ que dijo ser hablante de español, $n=5,803$ ). Cortez et al. (2017) reportan el desplazamiento del español en los adultos jóvenes que permanecieron en zonas en donde su uso se vio desfavorecido. Nava et al. (2017, pp. 125-136), se muestra que, a mayor edad de migración a los EUA, los estudiantes desarrollan un bilingüismo en español e inglés, conservando la lengua de origen (por uso y estudio formal en la escuela o fuera de ésta); sin embargo, los adultos bilingües jóvenes en la universidad, muestran un desbalance entre las habilidades de producción oral y las de lectoescritura en español, principalmente en los que su inglés predomina.

Santos et al. (2010) identificaron que tanto estudiantes con bilingüismo balanceado por la instrucción formal en modelos de inmersión dual como estudiantes con bilingüismo dominante en el idioma inglés, resultado del modelo de inmersión estructurada al inglés, han desarrollado la Competencia Lingüística/Transcultural en la L1 y L2. Sin embargo, Cortez et al. (2017) reportan que el efecto de un modelo de inmersión en el idioma inglés es el desplazamiento del español. Así, Camacho y Vargas (2017), Cortez et al. (2017), Nava et al. (2017) y Zúñiga (2013) apuntan a la necesidad de apoyar el desarrollo de la lectoescritura en español al retorno, pues el no haber desarrollado el español-desde el nivel básico al superior- los ha llevado incluso a la deserción escolar.

Autores como Camacho y Vargas (2017); Nava et al. (2017); Santos et al. (2010) y Smith (2006) también ofrecen algunas medidas para promover el desarrollo o mantenimiento del idioma inglés o español entre los retornados, dependiendo de la alta o baja concentración de estos estudiantes en el contexto, del igual o desigual dominio de los dos idiomas, la edad y nivel educativo al que ingresan. Así, para el nivel básico (preescolar y primaria) y en zonas de alta migración de retorno Smith (2006) considera que los niños con dominio del inglés podrían ser instruidos en aulas bilingües con modelos de inmersión/instrucción dual (con contenidos en ambos idiomas y el desarrollo de la biliteracidad). Por su parte, Santos et al. (2010) consideran aprovechable la capacidad excepcional en esta edad para adquirir la lengua local (el español u otro idioma). De manera alternativa, el mantenimiento de ambos idiomas lo constituyen el uso del inglés en ámbitos como el de la casa, y la instrucción en español en la escuela. Sin embargo, si cuando al retorno -y al ingreso a los distintos niveles educativos subsecuentes predomina el inglés-es necesario implementar medidas como un curso previo, propedéutico, clases extra de español o atender el desarrollo de la lectoescritura académica fortaleciendo su desempeño escolar (Camacho y Vargas, 2017; Nava et al., 2017; Santos et al., 2010).

Smith (2006) considera que la formación docente en zonas de alta concentración de retornados y entre los profesores interesados debe incluir cursos de formación en conocimientos lingüísticos, culturales y en la adquisición y desarrollo de la L1/L2 (aspectos psicológicos y sociolingüísticos). Esto permitiría identificar a los estudiantes bilingües e implementar estrategias didácticas que ven el bilingüismo en los estudiantes como "fondos de saber" y un recurso en las aulas; sin embargo, la suya es una postura restrictiva para las elecciones propias de los jóvenes estudiantes de retorno porque sugiere formar a los jóvenes adultos retornados como docentes de inglés. Por último, Alvarado y Reyes (2017), Cortez et al. (2015, 2017), Galindo (2016), Nava et al. (2017) y Smith (2006) coinciden en que el conocimiento y manejo de distintas reglas lingüísticas y culturales es un recurso que permite a los estudiantes jóvenes de retorno desenvolverse de forma exitosa en el nuevo contexto escolar universitario, y otorga oportunidades de 
empleo, influyendo incluso en la decisión de su profesión. ${ }^{4}$

\subsection{Condiciones de contexto y currículo para la integración al nuevo entorno educativo}

Camacho y Vargas (2017) y Santos et al. (2010) identificaron las siguientes necesidades administrativas en las escuelas en regiones de alta migración y retorno: el conocimiento de directivos, padres de familia y estudiantes de los acuerdos y normas que la Secretaría de Educación Pública ha implementado para facilitar los procesos de registro o inscripción de los estudiantes; la falta de documentos para acreditar la identidad (actas de nacimiento y pasaporte) y cartas o boletas de transferencia de calificaciones, para la inscripción escolar y la revalidación de materias, así como pobres condiciones de infraestructura (sin equipo o materiales).

En cuanto al currículum, Jensen et al. (2017) indagan la calidad educativa en el aula del preescolar, en municipios de alta y menor concentración de retornados nacidos en EUA, respecto a dimensiones como soporte emocional, organización del salón y apoyo instruccional, destacando la falta de habilidades docentes para recuperar las experiencias y conocimientos previos de los estudiantes y relacionarlos con el contenido curricular, así como la poca interacción con ellos o su invisibilización. En el nivel secundaria, Santos et al. (2010), apuntan a la necesidad de fortalecer los conocimientos y habilidades de los docentes y estudiantes (retornados y no retornados) ante grupos multinivel.

La diversidad de niveles del dominio de los idiomas inglés y español en los distintos niveles educativos hace necesaria la evaluación diagnóstica al ingreso para determinar el nivel de bilingüismo de los estudiantes (oral y escrito) y el conocimiento de materias sustantivas ${ }^{5}$; para acciones compensatorias y adecuaciones curriculares hacia un currículum incluyente y diferenciado, donde contenidos de la cultura de EUA sean valorados, reconocidos e incluso estudiados, propiciando motivación y disminuyendo el fracaso escolar (Alvarado y Reyes, 2017; Cortez et al., 2015, 2017; Nava et al., 2017; Santos et al., 2010; Valdéz et al., 2018; Zúñiga, 2013). Por su parte, Huerta et al. (2017) recomiendan la tutoría entre iguales en un programa de formación de docentes de lenguas como estrategia pedagógica para aprovechar la diversidad lingüística y cultural de los alumnos (estudiantes de retorno, indígenas y hablantes monolingües de español), favoreciendo el aprendizaje del español y el inglés.

Respecto al rezago escolar, Vargas y Camacho (2015) encontraron que está sujeto a condiciones de retorno (contexto urbano o rural), y tiende a afectar a varones de retorno de mayor edad y los que hablan una lengua indígena. En los estados de la zona central de migración (Oaxaca incluido), se observó más el rezago o la inasistencia a la escuela. Sobre la convivencia escolar, Alvarado y Reyes (2017) apuntan a la necesidad de la educación para la convivencia, pues en bachillerato se reproducen acciones que reflejan un estilo de vida y una educación diferentes, que no son comprendidos por sus pares y generan procesos de exclusión y discriminación. La incidencia de violencia, el abandono y el bajo capital social de los estudiantes migrantes de retorno podrían mejorarse con la vinculación escuela, docentes y padres de familia (Alvarado y Reyes, 2017; Santos et al., 2010; Vargas y Camacho, 2015).

\section{Discusión y conclusiones}

Las definiciones y tipología de la migración de retorno han considerado variables como edad (salida y retorno), lugar de nacimiento, nacionalidad, estatus migratorio, tránsito entre países, etc., sugiriendo la diversidad de situaciones, contextos, relaciones sociales, experiencias educativas, uso de lenguas, etc., en las que los migrantes de retorno se desenvuelven (Aguilar, 2012, 2015; Cortez et al., 2015, 2017; Nava et al., 2017; Santos et al., 2010; Zúñiga, 2013). Las problemáticas y necesidades de estos estudiantes varían en relación con el nivel educativo al que ingresan, sus capacidades bilingües, edad y nivel de escolaridad, recursos económicos, redes sociales y el capital cultural-humano de los padres o familiares (Alvarado y

\footnotetext{
${ }^{4}$ Algunos los adultos mirantes desarrollan un bilingüismo incipiente en inglés, pues en ellos domina el español y una posible lengua de origen (Smith, 2006).

5 Zúñiga (2013) identifica la Historia, Geografía, Ciencias y Español como contenidos críticos en los retornados.
} 
Reyes, 2017; Santos et al., 2010; Vargas y Camacho, 2015).

En la administración escolar resulta importante contar con información clara y certera en el registro y los procesos de evaluación diagnóstica para identificar niveles de conocimientos y habilidades (orales y escritas) en español, inglés o una lengua indígena, así como de los conocimientos básicos generales con que cuentan, independientemente de las especificidades curriculares del país que retornan (Camacho y Vargas, 2017; Santos et al., 2010).

Por las expectativas de los estudiantes sobre el sistema escolar al que ingresan y el cambio inesperado de contexto o de situación económica es necesario implementar acciones de acogida y acompañamiento para disminuir los efectos emocionales del retorno, lo que contribuiría a disminuir el rezago y bajo rendimiento académico, una malsana convivencia e incluso la deserción escolar (Alvarado y Reyes, 2017; Galindo, 2016; Jensen et al., 2017). Las recomendaciones hacia el desarrollo y mantenimiento del bilingüismo de los estudiantes quizás son las de más controversia y requiere de un análisis adecuado de la factibilidad de su implementación generalizada; aunque Santos et al. (2010); Smith (2006); Valdéz et al. (2018) y Zúñiga (2013) sugieren tomar en cuenta grados de concentración de población en las diferentes regiones migratorias, así como la necesidad de formación docente sobre el fenómeno y campo disciplinar con aplicación al contexto educativo.

Si bien los estudiantes universitarios muestran la capacidad de resignificar las experiencias adversas y consideran su bilingüismo como un recurso para lograr sus metas (Cortez et al., 2015, 2017; Huerta et al., 2017; Nava et al., 2017), también es responsabilidad de las instituciones educativas informar y brindar las oportunidades de desarrollo académico y personal que les corresponde a los estudiantes, al ingreso a la institución y durante sus estudios, mediante apoyos económicos, la formación integral y el fortalecimiento de sus competencias lingüísticas, facilitando su integración al sistema escolar y social. Todas estas estrategias son generalizables al resto de la población estudiantil, con beneficios en sus procesos de aprendizaje, además de ser las recomendadas y esperadas para el resto de los estudiantes en el sistema educativo en México.

Sin embargo, se requieren estudios que indaguen aspectos específicos del bilingüismo individual y social de los estudiantes. Por un lado, es interesante investigar los procesos psicolingüísticos y del desarrollo de su bilingüismo, el mantenimiento de éste fuera y dentro del contexto escolar, la interacción y las prácticas lingüísticas de estos estudiantes con relación a su desempeño escolar e integración. Por el otro, es evidente la falta de estudios con docentes y padres de familia para conocer sus necesidades, experiencias, perspectivas y estrategias de enseñanza-aprendizaje para la comunicación, el desarrollo de la biliteracidad o el mantenimiento de las lenguas, de este modo se les incluiría como agentes en la búsqueda de posibles soluciones en el marco de las propuestas curriculares, como la educación intercultural o los de la educación inclusiva vigentes en México. Con una mirada a los otros, el alcance y propósitos investigativos incluirían no sólo la exploración y la descripción, sino la explicación y comprensión del fenómeno, buscando la implementación de cambios o soluciones que benefician a los alumnos no migrantes, quizás también bilingües dependiendo de la región y plurilingüismo en el contexto.

El bilingüismo de los estudiantes de retorno resulta de especial importancia en el contexto educativo, ya que de ello depende su integración en el contexto escolar y las actividades académicas. La revisión de los estudios realizados contribuye a conocer las necesidades, en los diferentes niveles educativos y de distintas regiones migratorias en México, de los estudiantes de retorno, en donde su bilingüismo tiene un papel importante en su proceso de integración y formación escolar. Se distinguieron distintas variables individuales y sociales que promueven o afectan la integración, el desempeño académico, los procesos de comunicación, las trayectorias escolares y la convivencia de esta población en las instituciones educativas. Se identificaron posibles líneas de investigación respecto al bilingüismo de los estudiantes migrantes, como el desarrollo, mantenimiento o pérdida de éste, la relación biliteracidad-desempeño académico, así como la forma en la que los docentes y padres de familia abordan el fenómeno. 


\section{Referencias}

Aguilar, R. (2012). Migración de Menores de edad Estados Unidos-México en el período 2000-2010: ¿retornados o inmigrantes? Trabajo presentado Programa de Doctorado en Estudios de Población, Colegio de México.

Aguilar, R. (2015). Los niños y jóvenes en la migración de retorno a México. Una propuesta metodológica para su estudio. En F. Lozano y J. Martínez (Eds.), Retorno en los procesos migratorios de América Latina. Conceptos, debates, evidencias (pp. 243-265). ALAP Editor.

Alvarado, A. M. y Reyes, V. G. (2017). Convivencia escolar en el nivel medio superior. En V. G. Reyes y A. M. Alvarado (Coords.), La educación en México: escenarios y desafios (pp. 21-45). Juan Pablos Editor.

August, D. y Shanahan, T. (Eds.). (2006). Developing literacy in second-language learners. Report of the National Literacy Panel on Language-Minority Children and Youth [Desarrollando la lectoescritura en los aprendientes de una segunda lengua. Reporte del Panel Nacional de Literacidad en niños y jóvenes hablantes de lenguas minoritarias]. Lawrence Erlbaum Associates.

Baker, C. y Wright, W. E. (2017). Foundations of bilingual education and bilingualism [Fundamentos de educación bilingüe y bilingüismo]. Multilingual Matters.

Camacho, E. y Vargas, E. D. (2017). Incorporación escolar de estudiantes provenientes de Estados Unidos de América en Baja California, México. Sinéctica, (48), 1-18.

https://sinectica.iteso.mx/index.php/SINECTICA/article/view/659

Canales, A. C. (2001). Determinantes sociodemográficas del retorno y asentamiento en la migración México-Estados Unidos. En E. Patiño y J. Castillo (Comp.), Trabajo y Migración (pp. 139-158). 2o. Congreso RNIU: Investigación urbana y regional. Balance y Perspectivas. México: Red de Investigación Urbana. A.CBUAP.

Cortez, N., Altamirano, A. y García, A. (2017). Ideologías, políticas y competencia lingüística: universitarios migrantes de retorno en Sonora. Sinéctica, (48), 1-14.

https://sinectica.iteso.mx/index.php/SINECTICA/article/view/666

Cortez, N., Altamirano, A. y García, A. (2015). Estudiantes migrantes de retorno en México. Estrategias emprendidas para acceder a una educación universitaria. Revista Mexicana de Investigación Educativa, 20(67), 1187-1208. https://www.comie.orq.mx/revista/v2018/rmie/index.php/nrmie/article/view/163

Edward, J. (2014). Bilingualism and multilingualism: some central concepts. En T. K. Bhatia y W. C. Ritchie (Eds.), The handbook of bilingualism and multilingualism (pp. 5-25). Wiley Blackwell.

Galindo, G. E. (2016). Returnees, language socialization and enculturation (Tesis de Licenciatura no publicada). Universidad Autónoma Benito Juárez de Oaxaca.

Gonzalez-Вагrera, A. (2015). More mexicans leaving than coming to the U.S. Pew Research Center. https://www.pewresearch.org/hispanic/2015/11/19/more-mexicans-leaving-than-coming-to-the-u-s/

Goto-Butler, Y. (2014). Bilingualism/multilingualism and second language acquisition. The bilingual child. En T. K. Bhatia y W. C. Ritchie (Eds.), The handbook of bilingualism and multilingualism (pp. 109-136). Wiley Blackwell.

Hernández, R., Fernández-Collado, C. y Baptista, P. (2006). Metodología de la Investigación. McGrawHill Interamericana. 
Huerta, V., López, M. E., León, E. N. y Sughrua, W. M. (2017). Aprovechamiento de la diversidad lingüística y cultural a través de la tutoría entre colegas. En F. J. Murillo (Coord.), Actas del 11 Congreso Internacional sobre Liderazgo y Mejora de la Educación, RILME (83-86).

http://rinace.net/Avances en Democracia y Liderazgo Distribuido.pdf

Jensen, B., Mejía, R. y Aguilar, R. (2017). Equitable teaching for returnee children in México. Sinéctica, (48), 1-20. https://sinectica.iteso.mx/index.php/SINECTICA/article/view/757

Nava, R., Domínguez, R. y Castro, M. C. (2017). Jóvenes universitarios repatriados: retos y oportunidades en el área de lecto-escritura. Paradigma, 38(2), 121-139.

http://revistaparadigma.online/ojs/index.php/paradigma/article/view/624

Santos, J., Ibarra M., Carregha, L., de la Vega, M. y González, J. L. (2010). Diagnóstico de necesidades educativas de menores migrantes de retorno en San Luis Potosí.

https://studylib.es/doc/6002152/diagn\%C3\%B3stico-de-necesidades-educativas-de-menores-migrantes

Serratrice, L. (2014). The bilingual child. En T. K. Bhatia y W. C. Ritchie (Ed.), The handbook of bilingualism and multilingualism (pp. 87-108). Wiley Blackwell

Smith, P. H. (2006). Trasnacionalismo, bilingüismo y planificación del lenguaje en contextos mexicanos. En R. Terborg y L. García (Comps.), Retos de la planificación del lenguaje en el siglo XXI (Vol. II). UNAM. http://www.potowski.org/sites/potowski.org/files/Smith 2006.pdf

Valdéz, G. C., Ruiz, L. F., Rivera, O. B., López y R. A. (2018). Menores migrantes de retorno: problemática académica y proceso administrativo en el sistema escolar sonorense. Región y Sociedad, 30(72), 1-30. https://doi.org/10.22198/rys.2018.72.a904

Vargas, E. D. y Camacho, E. (2015). ¿Cambiarse de escuela? Inasistencia y rezago escolar de los niños de migración reciente de Estados Unidos a México. Norteamérica, Revista Académica del CISAN-UNAM, 10(2), 130. https://doi.org/10.20999/nam.2015.b006

Wei, L. (2014). Conceptual and methodological issues in bilingualism and multilingualism research. En T. K. Bhatia y W. C. Ritchie (Eds.), The handbook of bilingualism and multilingualism (pp. 26-51). Wiley Blackwell.

Zúñiga, V. (2013). Migrantes internacionales en las escuelas mexicanas: desafíos actuales y futuros de política educativa. Sinéctica, (40), 1-12. https://sinectica.iteso.mx/index.php/SINECTICA/article/view/50 